\title{
Inertial shear flow of assemblies of frictionless polygons: Rheology and microstructure
}

\author{
Émilien Azéma ${ }^{1, a}$, Farhang Radjaï ${ }^{1,2}$, and Jean-Noël Roux ${ }^{3}$ \\ 1 Laboratoire de Mécanique et Génie Civil (LMGC), Université de Montpellier, CNRS, Montpellier, France \\ 2 MSE2, UMI 3466 CNRS-MIT, CEE, Massachusetts Institute of Technology, 77 Massachusetts Avenue, \\ Cambridge 02139, MA, USA \\ 3 Université Paris-Est, Laboratoire Navier ${ }^{\mathrm{b}}$, 2 Allée Kepler, Cité Descartes, 77420 Champs-sur-Marne, France
}

\begin{abstract}
Motivated by the understanding of shape effects in granular materials, we numerically investigate the macroscopic and microstructural properties of anisotropic dense assemblies of frictionless polydisperse rigid pentagons in shear flow, and compare them with similar systems of disks. Once subjected to large cumulative shear strains their rheology and microstructure are investigated in uniform steady states, depending on inertial number $I$, which ranges from the quasistatic limit $\left(I \sim 10^{-5}\right)$ to 0.2 . In the quasistatic limit both systems are devoid of Reynolds dilatancy, i.e., flow at their random close packing density. Both macroscopic friction angle $\varphi$, an increasing function of $I$, and solid fraction $\nu$, a decreasing function of $I$, are larger with pentagons than with disks at small $I$, but the differences decline for larger $I$ and, remark-ably, nearly vanish for $I \sim 0.2$. Under growing $I$, the depletion of contact networks is considerably slower with pentagons, in which increasingly anisotropic, but still well-connected force-transmitting structures are maintained throughout the studied range. Whereas contact anisotropy and force anisotropy contribute nearly equally to the shear strength in disk assemblies, the latter effect dominates with pentagons at small $I$, while the former takes over for $I$ of the order of $10^{-2}$. The size of clusters of grains in side-to-side contact, typically comprising more than 10 pentagons in the quasistatic limit, very gradually decreases for growing $I$.
\end{abstract}

\section{Introduction}

Disordered assemblies of frictionless particles are the arche-typal representation of some amorphous materials made of athermal particles with very short-range interactions, such as suspensions and colloidal glasses. They have often been regarded as "reference systems" that highlight generic features of granular materials. In the absence of friction, the particles are allowed to reach their lowest configurational energy without being trapped in metastable states as a consequence of frictional frustration. If subjected to isotropic confining forces (under hydrostatic pressure) their volume should be minimized. Such isotropically confined configurations therefore coincide with the so-called "random close packing" (RCP) states - densest possible disordered arrangements of rigid, impenetrable

\footnotetext{
${ }^{a}$ e-mail: emilien.azema@umontpellier.fr

b Laboratoire Navier (UMR8205) is a joint laboratory, depending on École Nationale des Ponts et Chaussées, Institut Français des Sciences et Technologies des Transports, de l'Aménagement et des Réseaux and Centre National de la Recherche Scientifique.
}

grains. The interest in this classical concept [1,2], was thus recently revived, and, over the past 20 years RCP states have been quite intensively investigated and discussed, primarily for identical spherical grains [3-5], then for various grain shapes: ellipsoids with different aspect ratios [6-10], monodisperse Platonic or Archimedian polyhedra [11-13], "superballs" [14] (i.e., smooth objects with varying shapes interpolating continuously from cubes to octahedra) ... RCP configurations, prepared without friction, were also used as maximally dense initial states in the simulation of shear tests with frictional irregular polyhedra with increasing angularity [15], or with nonconvex grains [16]. Among the issues repeatedly addressed in studies of isotropically assembled packs of frictionless objects are the uniqueness, or lack thereof, of "the RCP state" $[5,17,18]$; and the absence of force indeterminacy $[3,5]$, which may or not, according to the stability of "floppy modes" [19,20], imply the isostaticity of the forcecarrying structure, and which entails a singular distribution of the (anomalously soft) eigenmodes of the stiffness matrix [21].

The rheology of assemblies of frictionless, rigid objects of different shapes has, in comparison, elicited 
considerably fewer works. Studies of quasistatically deformed assemblies of grains of varying shapes [10, 22-27], or of their flow in the inertial regime [28-30] usually introduce intergranular friction, which, in addition to geometric features, essentially determines the material behaviour, outside a small strain regime [31] — the limited effects of restitution coefficients in collisions (or viscous dissipation), which vanish in the quasistatic limit, are often negligible in the dense inertial flow regime $[28,31]$. The material ability to resist shear stems from friction and from geometric effects associated to steric exclusion [32]. In the absence of friction, the material behaviour essentially results from the geometry of solid grain packs. The contact networks, due to the lack of force indeterminacy of frictionless rigid grain assemblies, are fragile and tend to become exquisitely sensitive to variations in the applied load $[33,34]$. Their ability to adapt to stress variations and their rearrangement patterns are at the microscopic origin of some basic and mysterious properties of rigid, frictionless grains, as recently documented for spherical beads [34,35], as well as for disks and pentagons [36-39] in two dimensions (2D): granular materials made of such particles possess some macroscopic (internal) friction coefficient, but are devoid of dilatancy. These properties are remarkable in several respects. The absence of dilatancy, in particular, contradicts ideas dating back to Reynolds [40], who coined the word "dilatancy" and attributed the phenomenon to steric exclusion effects. While the behaviour of frictionless grains is entirely dominated by steric exclusion, there is no dilatancy in that case. Furthermore, the qualitative similarity between rounded and angular particles (which could be naively be expected to behave like frictional ones) also seems counter-intuitive, given the observed rheological influence of side-to-side contacts between polygons $[15,41,42]$.

The objective of the present paper is to further investigate the connections between macroscopic rheological properties, in quasistatic or inertial shear flow, of assemblies of rigid frictionless $2 \mathrm{D}$ objects, with systematic comparisons between disks and pentagons. It is an extension of ref. [36], (a short paper published as a "rapid communication") which focussed on internal friction and solid fraction in the quasistatic limit. Compared to this previous publication, a wider range of inertial numbers (the reduced shear rate that characterizes the state of the flowing material) is explored, away from the quasistatic limit, and inertial effects on disks and on pentagons are compared. Contact networks are characterized in greater detail, distinguishing the peculiar role of side-side contacts in the case of pentagons, with some observations on their spatial organization in the shear flow. Unlike previous studies of shape effects [42-44], the present one, by dealing with frictionless objects, focusses on geometry alone.

The model system and the simulation procedures are presented in sect. 2. Section 3 focusses on shear strength, macroscopic stresses and solid fraction for different values of the inertial number. The microstructure is analyzed in sect. 4 in terms of particle connectivity, fabric and force anisotropies, and role of side-side contacts. We conclude with a summary of the main findings of this work.

\section{Model description}

\subsection{Numerical procedures}

The simulations are carried out by means of the contact dynamics (CD) method, which, as described in a number of publications [45-49], is based on an implicit time integration of the equations of motion and on a nonsmooth formulation of mutual exclusion between particles (as well as a non-smooth formulation of the Coulomb condition, if the grains are frictional). Up to small numerical errors, thresholds are sharply defined in the method, which assumes the grains to be perfectly rigid. As a result, the simulations can be carried out with large time steps compared to the more usual molecular dynamics (MD) approach, also referred to as a discrete element method (DEM) with deformable grains, which considers elastic contacts $[49,50]$. The contacts are identified from the tiny overlaps of neighboring grains, which are calculated, for each pair, through the so-called shadow overlap method [51]. In the case of pentagons, contacts are of two types: side-vertex contacts (1), and side-side (2) ones (the approach to vertex-vertex ones, of little statistical relevance, is dealt with as in ref. [42]). Side-vertex contacts are "simple" contacts, similar to the contacts between disks. They introduce one unilateral constraint between two objects, and the normal vector, which carries the repulsive force or momentum transfer in the absence of friction, is perpendicular to the side. Side-side contact are "double" contacts in the sense that they can be represented by two unilateral constraints. A double contact is equivalent to a pair of simple contacts between the same pentagons, and the normal direction is the normal to their common side. In practice, two forces are calculated at each side-side contact, but only the resulting net force and torque are physically meaningful. The CD method has been extensively employed in two and three dimensions for the simulation of granular materials with various shapes: disks of varying polydispersity $[22,52,53]$, elongated $[10,54]$ or nonconvex [55] 2D objects, various $3 \mathrm{D}$ polyhedra subjected to compression $[15,56]$ or in dense flow [57], non-convex $3 \mathrm{D}$ objects $[16]$.

\subsection{Simple shear test}

The system simulated is actually the same as in ref. [36]. We generate numerical samples composed of 15000 grains, pentagons or disks. Particle sizes are randomly chosen according to a uniform distribution in surface area, the diameter $d$ of the circumscribed circle varying between $D_{\min }$ and $D_{\max }=2 D_{\min }=\langle d\rangle / \ln (2),\langle d\rangle$ denoting the average value of $d$.

The particles are initially placed in a cell of width $200\langle d\rangle$ along the $x$-axis, using a geometrical procedure [58, 59]. The grains belonging to the bottom (lowest $y$ coordinates) and top (highest $y$ ) layers, of thickness $3\langle d\rangle$ are glued together, forming parallel rough walls. The cell height $H$ is defined as the distance between the $y$ coordinate of the centers of mass of both walls. While the 


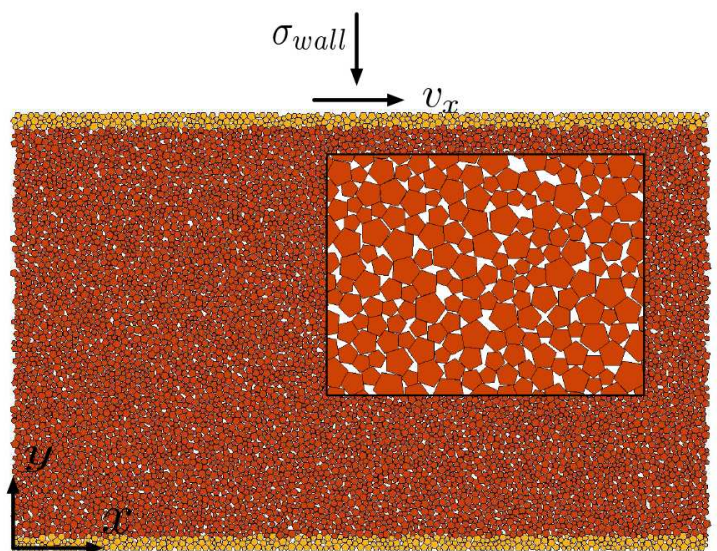

Fig. 1. (Colour online) Plane shear simulation. Yellow (light gray) particles constitute the rough walls. A zoom is shown in the inset.

bottom wall (small $y$ ) is maintained fixed, a shear flow is imposed by ascribing a constant lateral velocity $v_{x}$ to the upper wall. Its $y$ coordinate might fluctuate, in order to maintain a constant confining stress $\sigma_{y y}$ in the velocity gradient direction, as shown in fig. 1. To avoid strain localization near the walls, the grains in contact with the upper and lower plane walls are "glued" to them. The resulting wall roughness insures a nearly uniform distribution of strain rate in the sample. The system is free of gravity. Grain interactions are characterized by three parameters: the coefficient of friction (maintained at zero here), and the coefficients of normal and tangential restitution, which control the rate of dissipation in collisions. Those coefficients are set to zero here. The quasistatic behaviour (see below) of sheared granular systems is independent of the coefficient of restitution unless this parameter is set to very large values (above 0.8) [60].

The samples are sheared up to a large cumulative shear strain $\gamma=\Delta x / H$, defined as the ratio of upper wall displacement $\Delta x$ is the flow direction $x$ to the (average) sample height $H$. Assuming homogeneous steady states are observed with shear rate $\dot{\gamma}=\frac{v_{x}}{H}$ and normal stress $\sigma_{y y}$, dimensional analysis requires that dimensionless forms of macroscopic constitutive equations and of internal state parameters only depend on the inertial parameter $I$, defined as $[28,31,60]$

$$
I=\dot{\gamma}\langle d\rangle \sqrt{\frac{\rho_{m}}{\sigma_{y y}}}
$$

$\rho_{m}$ denoting the mass density of the solid material which the grain are made of. The inertial number characterizes the importance of inertial effects in dense granular flows (for which it has played a central role in the design of successful constitutive laws [61-63]), and the quasistatic limit is the limit of $I \rightarrow 0$. The simulations reported here are carried out with $I$ values ranging from $4.710^{-4}$ to 0.25 for pentagons and from $6.210^{-6}$ to 0.20 for discs. A total number of 32 shear flow configurations are simulated, for a total cumulative shear strain ranging from $\gamma=4$ (for $I=3.10^{-4}$ ) to $\gamma=50$ (for $I=0.25$ ). We consider

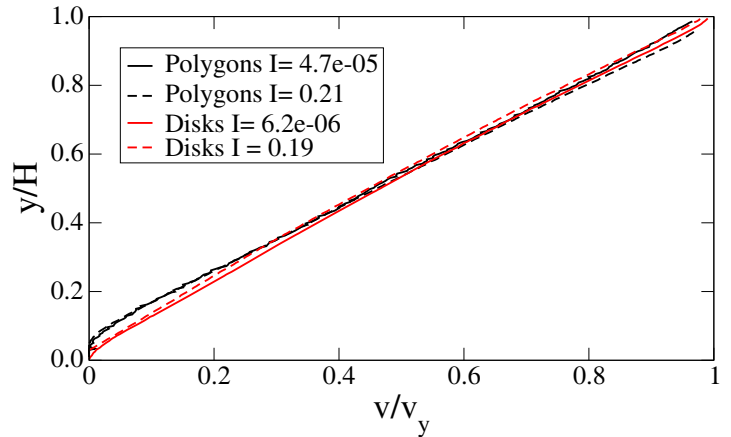

Fig. 2. (Colour online) Time-averaged velocity profiles in disk and pentagon samples for small and large values of $I$.

that the permanent shear state is reached if the relative fluctuations of the measured quantities are small enough, typically from $\gamma>1$ for $I=3.10^{-4}$, and $\gamma>30$ for $I=0.25$

As shown in fig. 2, the sample is homogeneously sheared throughout the range of inertial numbers. Velocity profiles exhibit a small systematic wall effect, especially with pentagons, affecting thin layers adjacent to the walls. Outside these boundary layers, the shear rate, albeit a little different from its nominal value $v_{x} / H$, remains uniform through the major part of the sample. Likewise, the solid fraction and the stress components are constant in the bulk.

In the following all data are average values over the steady state with standard deviations used as error bars. The fluctuations increase with $I$, and thus the data are to be sampled more frequently in the steady state in order to reach meaningful statistics.

\section{Macroscopic constitutive relations}

\subsection{Stresses and friction coefficient}

We use the usual formula [64] for stress component $\sigma_{\alpha \beta}$, averaged over the sample, of surface area $A . \sigma_{\alpha \beta}$ relates to contact forces (force $\mathbf{F}_{i j}$ denotes the force transmitted by grain $i$ to its contacting neighbor $j$ ), to contact branch vectors $\mathbf{r}_{i j}$ (vector $\mathbf{r}_{i j}$ points from the centre of $i$ to the centre of $j-$ or, rather, the centre of the nearest image of $j$ by the translations along axis $x$ associated to the boundary conditions), and to grain masses $m_{i}$ and velocities $v_{i}$, as

$$
\sigma_{\alpha \beta}=\frac{1}{A}\left[\sum_{i<j} F_{i j}^{\alpha} r_{i j}^{\beta}+\sum_{i} m_{i} v_{i}^{\alpha} v_{i}^{\beta}\right] .
$$

In (2), the second, kinetic term, involving velocity fluctuations $^{1}$, remains very small compared to the first one (the sum over all contacts $i, j)$ in all the simulations considered here.

${ }^{1} v_{i}$ in (2) should actually denote the difference between the grain velocity and the local value of the macroscopic velocity, if the formula is to give the value of the Cauchy stress tensor. 
The macroscopic friction coefficient, $\mu$, is most usually defined as the ratio of the shear stress to the normal stress inside the material [63], and is associated to the friction angle, $\varphi$, as defined according to the Mohr-Coulomb criterion, as follows:

$$
\mu=\frac{\left|\sigma_{x y}\right|}{\sigma_{y y}}=\tan \varphi
$$

An alternative expression of the friction angle involves the principal stresses (eigenvalues of the stress tensor) $\sigma_{1}$ and $\sigma_{2}<\sigma_{1}$, combined into the average pressure, $p=\left(\sigma_{1}+\right.$ $\left.\sigma_{2}\right) / 2$ and the deviator stress $q=\left(\sigma_{1}-\sigma_{2}\right) / 2$, and reads

$$
\sin \varphi^{*}=\frac{q}{p}
$$

For a material exactly abiding by the Mohr-Coulomb failure criterion, both definitions of the friction angle would exactly coincide, $\varphi=\varphi^{*}$. However, granular materials in general [63], and assemblies of frictionless grains in particular [34], do not exactly satisfy the Mohr-Coulomb conditions, and the apparent angle of friction slightly differs according to the type of flow (e.g., simple shear compared to biaxial compression or "pure shear"). A normal stress difference $\sigma_{x x}-\sigma_{y y}$ in shear flow is observed in general, although sometimes reported to be negligible [63]. Normalizing it by the controlled stress $\sigma_{y y}$, and introducing the notation

$$
\Delta=\frac{\sigma_{x x}-\sigma_{y y}}{2 \sigma_{y y}}
$$

the values of the principal stresses, $\sigma_{1}$ and $\sigma_{2}$ are readily obtained on diagonalising the stress matrix, as

$$
\begin{aligned}
& \sigma_{1}=\sigma_{y y}\left[1+\Delta+\sqrt{\tan ^{2} \varphi+\Delta^{2}}\right] \\
& \sigma_{2}=\sigma_{y y}\left[1+\Delta-\sqrt{\tan ^{2} \varphi+\Delta^{2}}\right] .
\end{aligned}
$$

The corresponding principal directions are respectively oriented with angles $\theta_{1}$ and $\theta_{2}$ with respect to the flow direction, with

$$
\theta_{1}=\pi-\arctan \frac{\sqrt{\tan ^{2} \varphi+\Delta^{2}}-\Delta}{\tan \varphi} ; \quad \theta_{2}=\theta_{1}-\frac{\pi}{2} .
$$

From (6) and (4), $\varphi$ and $\varphi^{*}$ are related as

$$
\sin ^{2} \varphi^{*}=\frac{\tan ^{2} \varphi+\Delta^{2}}{(1+\Delta)^{2}}
$$

As a consequence of (6) and (7), a Mohr-Coulomb material with $\varphi=\varphi^{*}$ would satisfy $\Delta=\tan ^{2} \varphi$ and $\theta_{1}=\frac{3 \pi}{4}+$ $\frac{\varphi}{2}$ [63]. Another remarkable case [63], is the material for which $\Delta$ might be neglected (very small values are indeed reported in the literature [28]). Then, even though $\varphi$ and $\varphi^{*}$ are different, the same friction coefficient is identified from either approach, as relation (8) results in $\tan \varphi=$ $\sin \varphi^{*}$. In such a case the principal stress directions would

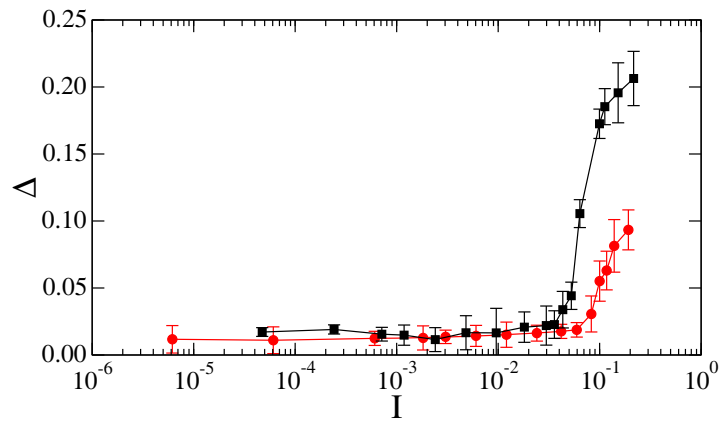

Fig. 3. (Colour online) Relative normal stress difference $\Delta$ (eq. (5)) versus $I$, for pentagons (black squares) and disks (red circles).

be inclined at angles $\theta_{1}=3 \pi / 4$ and $\theta_{2}=\pi / 4$ with respect to the flow direction, just like in a Newtonian viscous fluid.

In the present simulations the variations of $\Delta$ with $I$ are shown in fig. 3. In the quasistatic limit of small $I$ [36], relations (3) and (4) define about the same friction angle. One gets the following values for the quasistatic limit $\mu_{0}$ of the macroscopic friction coefficient defined as $\mu=\tan \varphi$ :

$$
\begin{array}{lr}
\mu_{0}=0.100 \pm 0.002 & (\text { disks) }, \\
\mu_{0}=0.180 \pm 0.002 & \text { (pentagons) }
\end{array}
$$

while the $I \rightarrow 0$ limits of $\sin \varphi^{*}$ are $0.099 \pm 0.02$ for disks and $0.183 \pm 0.002$ for pentagons. This corresponds to friction angles $\varphi \simeq \varphi^{*}=5.7 \pm 0.12^{\circ}$ for disks, while $\varphi=10.2 \pm 0.1^{\circ}$, differs very slightly from $\varphi^{*}=10.5 \pm 0.2^{\circ}$ for pentagons.

Away from the quasistatic limit, $\Delta$ strongly increases in the range $I \sim 10^{-2}$ and reaches values near 0.2 for pentagons. One should therefore distinguish between both definitions in the inertial regime, and we adopt the definition of the friction coefficient and the friction angle used in relation (3) throughout the following. Angles $\varphi, \varphi^{*}$, as well as $\theta_{1}$ (compared to the Mohr-Coulomb prediction), are given in table 1 for disks and for pentagons at the smallest and the largest investigated $I$ values.

Internal friction coefficient $\mu=\tan \varphi$ is plotted versus $I$ for disks and pentagons in fig. 4 . The increase of $\mu$ with $I$ is a familiar feature of granular rheology [28-30,35,61]. It is somewhat faster for disks than for pentagons, so that, although initially smaller, the friction coefficient of disks becomes similar to the one of pentagons as $I$ reaches $0.15-0.2$. Variations of $\mu$ with $I$ are most often modeled as power functions $[28,29,35,38]$, although the exponent might depend on the chosen interval of $I$ over which the power law function is fitted to the data:

$$
\mu=\mu_{0}+a I^{\alpha}
$$

While $\mu_{0}$ values are given in (9), exponent $\alpha=0.52$ (as already suggested for disks in two dimensions [65] and low $I$ values) turns out to adequately describe our data with both types of particles through the entire investigated range of $I$, with $a \simeq 0.54$ for disks and $a \simeq 0.39$ for pentagons. Our results differ slightly from those of Vågberg 
Table 1. Values of angles $\varphi, \varphi^{*}, \theta_{1}$ and relative normal stress difference $\Delta$, for the smallest and highest values of $I$ in packings of disks and pentagons.

\begin{tabular}{ccccccc}
\hline Samples & $I$ & $\varphi$ & $\varphi^{*}$ & $\Delta$ & $\theta_{1}$ (eq. (7)) & $\theta_{1}$ from Mohr Coulomb \\
\hline Disks & $6.210^{-6}$ & $5.7 \pm 0.12^{\circ}$ & $5.7 \pm 0.12^{\circ}$ & 0.02 & $140.7^{\circ}$ & $137.9^{\circ}$ \\
Pentagons & $4.710^{-5}$ & $10.2 \pm 0.10^{\circ}$ & $10.5 \pm 0.2^{\circ}$ & 0.025 & $138.9^{\circ}$ & $140.1^{\circ}$ \\
Disks & 0.19 & $17.1 \pm 0.10^{\circ}$ & $17.7 \pm 0.10^{\circ}$ & 0.095 & $143.3^{\circ}$ & $143.9^{\circ}$ \\
Pentagons & 0.21 & $19.7 \pm 0.13^{\circ}$ & $20.1 \pm 0.13^{\circ}$ & 0.20 & $149.5^{\circ}$ & $144.9^{\circ}$ \\
\hline
\end{tabular}

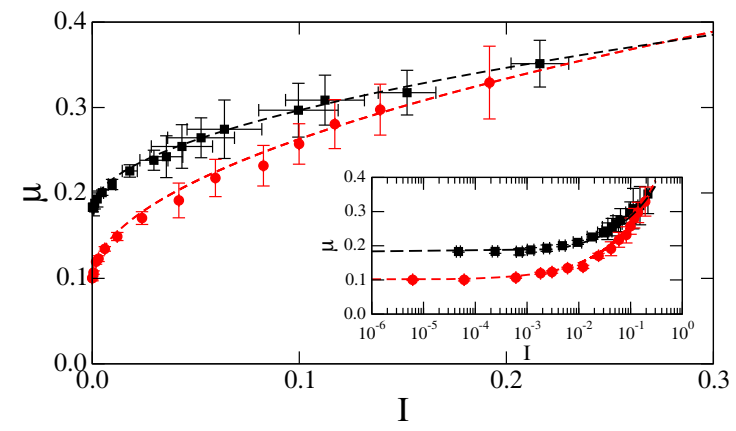

Fig. 4. (Colour online) Internal friction coefficient, as defined in (5), versus $I$, for pentagons (black squares) and disks (red circles). Inset: log-linear plot. Fits through data points with eq. (10) are shown as dotted lines.

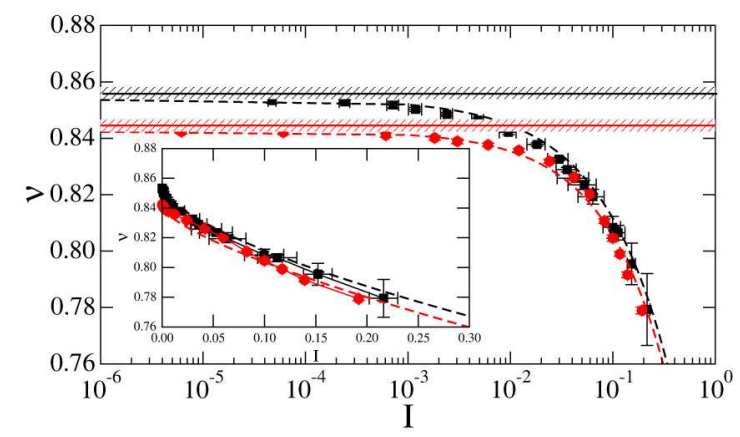

Fig. 5. (Colour online) Solid fraction $\nu$ versus $I$ for pentagons (black squares) and disks (red circles), on a log-linear plot. Inset: linear plot. Hashed zones about horizontal lines indicate $\mathrm{RCP}$ values of $\nu$, with their error bar.

et al., who carried out extensive and accurate simulations of bidisperse assemblies of disks under shear flow $[38,66]$ and found $\mu_{0} \simeq 0.093$ and $\alpha \simeq 0.4$ (see footnote ${ }^{2}$ ).

\subsection{Solid fraction}

The solid fraction, $\nu$, defined as usual as the fraction of the system area occupied by the particles, varies with inertial number as shown in fig. 5 . As often observed too $[28,35$,

\footnotetext{
${ }^{2}$ It should be noted that these authors warned that the "true" exponents, which they relate to the criticality of the jamming transition, are very likely hard to capture in twodimensional systems. We do not claim to track accurate critical exponents in the present study, which focusses on the differences between pentagons and disks.
}

36], $\nu$ is a decreasing function of $I$, which may also be fitted by a power law

$$
\nu=\nu_{0}-b I^{\beta}
$$

al least within some interval of $I$.

In the quasistatic limit of $I \rightarrow 0, \nu_{0}$ was observed [36] to coincide with the RCP value, meaning that assemblies of frictionless disks or pentagons are devoid of dilatancy. They do not change in surface area while deforming as the applied stress evolves from an isotropic pressure to a deviatoric state large enough to fulfill the yield condition. This quasistatic value is larger for pentagons, with $\nu_{0}=0.854 \pm 0.003$ than for disks, for which we find $\nu_{0}=0.8425 \pm 0.0008$ (compatible with the value given in ref. [38], despite the different diameter distribution). For growing $I$ the decrease in $\nu$ is slower in assemblies of disks, so that for larger $I$ values $\left(I \geq 10^{-2}\right)$ both materials have very similar densities. Our data are correctly adjusted over the whole $I$ range with $b=0.19$ and an exponent that could be slightly larger for disks $(\beta=0.71)$ than for pentagons $(\beta=0.65)$, although the difference is probably not meaningful. (The fit proposed in ref. [65] for disks, with a different polydispersity, with $\beta=0.69$ and $b=0.213$ is adequate for $I \leq 10^{-2}$.)

In the following we analyze the $I$-dependent microstructural properties of our packings and their possible connections with macroscopic stresses and density.

\section{Microstructural and micromechanical aspects}

The internal structure of the flowing material, which is basically controlled by steric exclusions and force transmission conditions [32], can be described in terms of various statistical descriptors pertaining to the force-bearing network of particles, as investigated in this section: particle connectivity (sect. 4.1) force and fabric anisotropies (sect. 4.2), specific role of "double" contacts between pentagon sides (sect. 4.3). Correlations are pointed out with the macroscopic behaviour and its $I$-dependence.

\subsection{Particle connectivity}

Figures 6 shows snapshots of the networks of forcecarrying contacts in the steady state for the disc (a) and pentagons (b) packing at large values of $I$. As observed in 


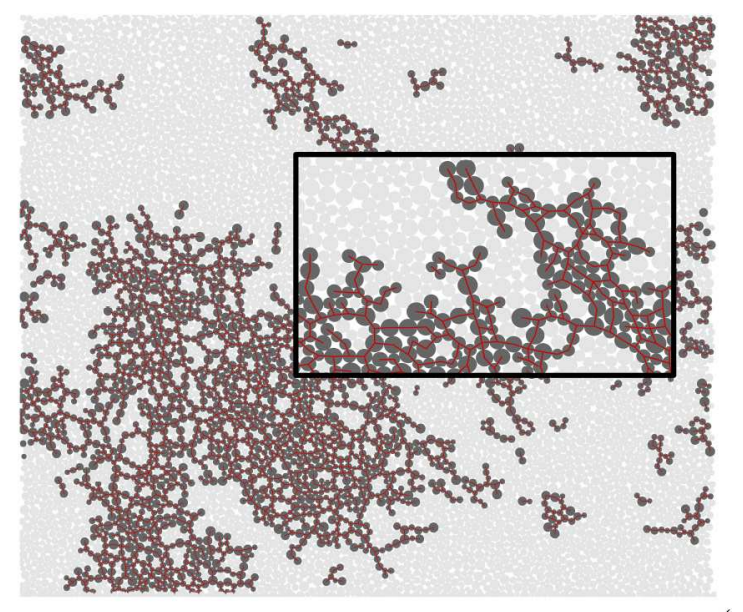

(a)

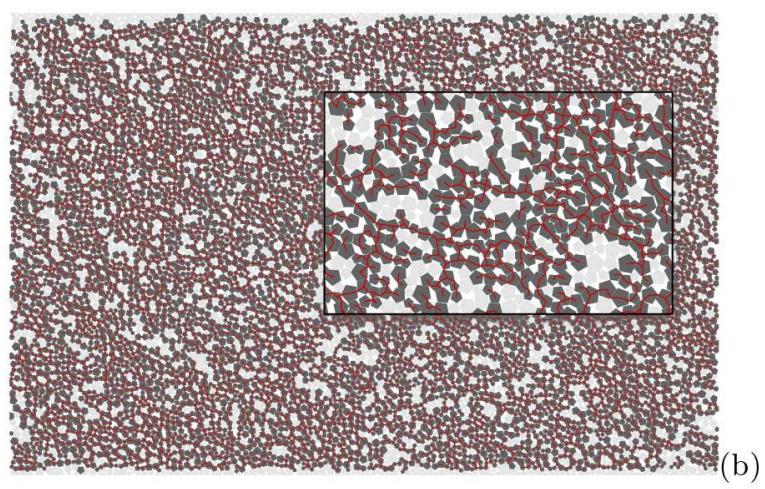

Fig. 6. (Colour online) Contact networks in disk (a) and pentagons (b) samples for $I \simeq 0.1$ and $I \simeq 0.25$, respectively. Rattlers (i.e., particles with no contact) are drawn in light gray. Red lines join grain centres of mass to contact points. A zoom is shown in the inset.

a number of earlier studies $[28,30,35]$ the contact network topology strongly varies with $I$, with quite a fast decrease of its density (and of the coordination number) between the quasistatic limit and the strongly inertial regime at $I \sim 0.1$. Floating particles, carrying no load (often referred to as the rattlers), are rare and isolated in quasistatic flow, while they tend to form large "fluidized zones" [30] for the highest $I$ values. However, the depletion of the contact network for growing $I$ is significantly faster and stronger for disks than for pentagons.

Quantitatively, the particle connectivity can be analyzed by the set of proportions $P_{c}$ of non-rattler grains having exactly $c$ contacting neighbors. $P_{c}$ values are plotted as functions of $I$ in fig. 7 . For pentagons $P_{0}$ increases with $I$ from nearly 0.1 , and tends to saturates at 0.25 at larger values. This contrasts with the increase of $P_{0}$ in disk assemblies, for which the fluidized zones tend to coalesce and form larger and larger regions, spanning the entire sample (fig. 6), as observed with frictional beads in $3 \mathrm{D}$ [30]. For $I<10^{-3}$, due to inertia effects, the fraction of highly connected pentagons $(c \geq 4)$ is reduced, as low-connected ones $(c \leq 3)$ become more numerous, and $P_{4}$ remains nearly constant. For larger $I$ values,
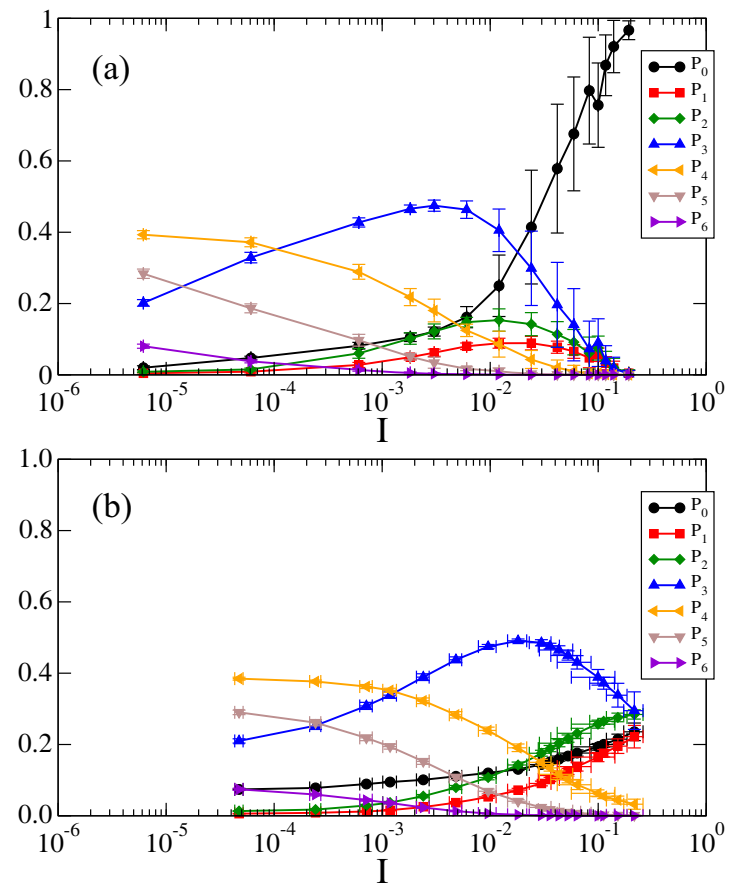

Fig. 7. (Colour online) Connectivity $P_{c}$ of the particles as a function of $I$ in log-linear scale: (a) for discs and (b) for pentagons.

$P_{4}$ declines whereas $P_{3}$ increases to a maximum value near $I \simeq 10^{-2}$ before beyond which $P_{3}$ declines also. This trend is similar to that of frictional spheres [30]. In contrast, $P_{1}$ and $P_{2}$ increase with $I$ but tend to saturate to 0.2 and 0.3 , respectively, meaning that the flows is not dominate by binary shocks as it is the case for rapid flows of frictional beads [30]. Nevertheless, the loss of particles having 3 contacts is a dramatic change in the microstructure as multiple contacts may thereafter occur in the form of linear chains without branching, as observed in fig. 6(b). The general evolution of the contact network for growing $I$ in the pentagon assembly is a gradual transition towards depleted networks, which remain connected over large length scales at $I=0.25$, and in which filamentary structures tend to emerge as the dominant morphological feature. The evolution of connectivity with $I$ is quite different with pentagons from the behavior of disk assemblies. Disks tend to lose more contacts at lower $I$ values, and the fast increase of the rattler fraction $P_{0}$ is quite a striking phenomenon, with only a small fraction of non-rattler grains remaining for $I \geq 0.1$. The maximum of $P_{3}$ is shifted to $I \simeq 0.003$, and there is no range of $I$ in which $P_{0}, P_{1}$ and $P_{2}$ simultaneously increase to significant values while $P_{3}$ decreases. The dilution of the contact network in disk systems for growing $I$ does not involve the filamentary morphology appearing for $I$ of order 0.1 in pentagon assemblies.

From $P_{c}$, we define the coordination number by $z=\sum_{c \geq 0} c P_{c}$. One may also define the coordination number of non-rattler grains, as $z^{*}=z /\left(1-P_{0}\right)$. For disks $z^{*}$ approaches the isostatic value of 4 in the quasistatic limit, 


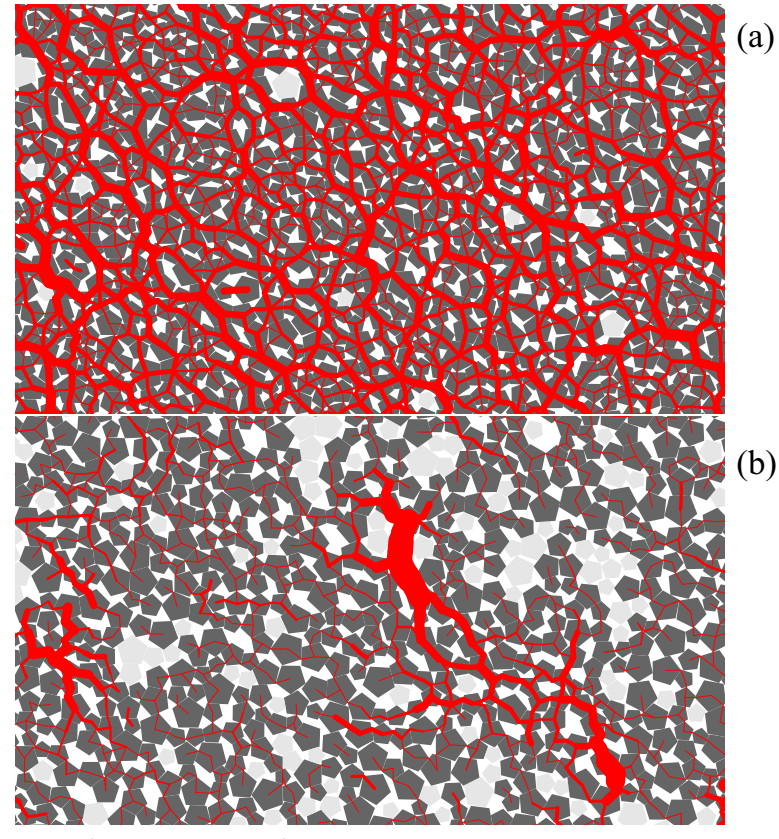

Fig. 8. (Colour online) Snapshots of the force network for two samples composed of pentagons, at $I \simeq 4.10^{-5}$ (a) and $I \simeq 0.25$ (b). The floating particles (i.e., particles with no contact) are drawn in light gray and the forces are represented by red line segments joining the centers of mass of the particles with the contact points. Line thickness is proportional to the contact force.

and decreases for growing $I$, as inertial effects disrupt the force-carrying network in shear flow. One thus observes $z^{*}=3.993$ with $I=6.10^{-6}$, decreasing to low values, near 0.05 at $I=0.2$. For pentagons, one should distinguish simple and double contacts, for which rattler-corrected coordination numbers, denoted respectively as $z_{1}^{*}$ and $z_{2}^{*}$, should satisfy $z_{1}^{*}+2 z_{2}^{*}=6$ in an isostatic network. We obtain $z_{1}^{*} \simeq 2.97$ and $z_{2}^{*} \simeq 1.50$ for the lowest value of $I$, showing that this relation is indeed approached in the quasistatic limit. It should be noted, however, that the model for pentagons has one non-generic feature enabling some force indeterminacy: pentagons are regular and angles between edges take well define values, adding up to multiples of $\pi$. Loops of side-to-side contacts are possible, and any such independent loop adds one to the degree of force indeterminacy ${ }^{3}$. Such loops occur rarely (see sect. 4.3) and do not appear to be statistically significant ${ }^{4}$.

This study of the evolution of the topology of the contact network should be supplemented with investigations into their orientations and forces, and information should also be supplied on their persistance and reorganization.

\footnotetext{
${ }^{3}$ A set of self-balanced forces is obtained if a doublet of equal and opposite normal forces is transmitted in each contact around the loop in such a way that torques balance on each grain.

${ }^{4}$ It is possible that the finite accuracy with which the kinematics of rigid pentagons is dealt with in the time-discretized Contact Dynamics approach contributes to eliminate some of these loops. They would not occur, anyway, in a generic system of pentagons with random angles.
}

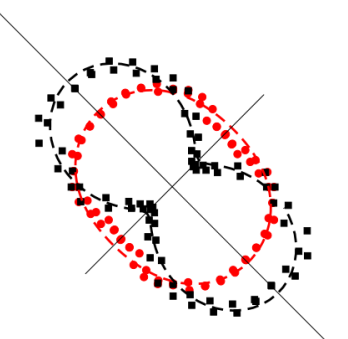

(a)

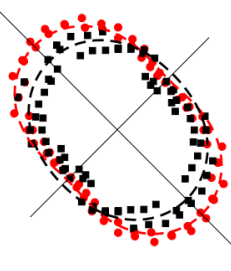

(b)

Fig. 9. (Colour online) Polar diagrams of $P_{\theta}(\theta)$ (a), and $\left\langle f_{n}\right\rangle(\theta)$ (b) for pentagons (black squares) and disks (red circles) together with their fits (dashed line) at large $I$.

\subsection{Force and fabric anisotropies}

The origins of macroscopic friction, which characterizes stress anisotropy, are to be found in anisotropic features of the force network. Figure 8 shows a typical map of forces in the steady shear flow of a pentagon sample for the lowest (a) and highest (b) values of $I$. Force chains are stronger but shorter for large $I$ values, and the large forces seem to be preferentially oriented near the compression direction, inclined at 45 degrees with respect to the flow direction. As in a number of previous studies, one may characterize the force network anisotropy in terms of parameters of the distribution of the angle $\theta$ between the flow direction and the contact normal vector $\boldsymbol{n}$; and of the $\theta$-dependent average intensity of the forces carried by contacts with orientation $\theta,\left\langle f_{n}\right\rangle(\theta)$. Let us recall that for "double" (side-side) contacts, the physically relevant force is the sum of the two forces used in the simulation. The ( $\pi$-periodic) probability density function $P(\theta)$ of contact normal orientations, and the ( $\pi$-periodic) function $\left\langle f_{n}\right\rangle(\theta)$ are statistical descriptors of the anisotropies of the contact network and of the force intensities. In a sheared granular material, these functions tend to take a simple unimodal shape, which can be well approximated by the lowest-order Fourier expansion $[10,15,67]$ :

$$
\left\{\begin{array}{l}
P(\theta)=\frac{1}{2 \pi}\left\{1+a_{c} \cos 2\left(\theta-\theta_{c}\right)\right\} \\
\left\langle f_{n}\right\rangle(\theta)=\left\langle f_{n}\right\rangle\left\{1+a_{n} \cos 2\left(\theta-\theta_{n}\right)\right\},
\end{array}\right.
$$

where $a_{c}$ is the contact orientation anisotropy, $a_{n}$ is the normal force anisotropy and $\left\langle f_{n}\right\rangle$ the mean normal force. Angles $\theta_{c}$ and $\theta_{n}$, with respect to the flow direction, indicate the directions that respectively maximise the density of contacts and the average force. These directions nearly coincide with the major principal stress direction $\theta_{1}$ in the sheared material, as illustrated in fig. 9. Specifically, $\theta_{c} \simeq \theta_{n}$ is near $136^{\circ}$ and $145.2^{\circ}$ for disks at small, respectively large, values of $I$, and $\theta_{c} \sim \theta_{n}=136.3^{\circ}$ and $146.3^{\circ}$ for pentagons at small, respectively large, values of $I$.

Parameters $a_{c}$ and $a_{n}$ can be estimated by fitting the measured values of $P_{\theta}(\theta)$ and $\left\langle f_{n}\right\rangle(\theta)$, respectively, but are more conveniently obtained from the following fabric and 


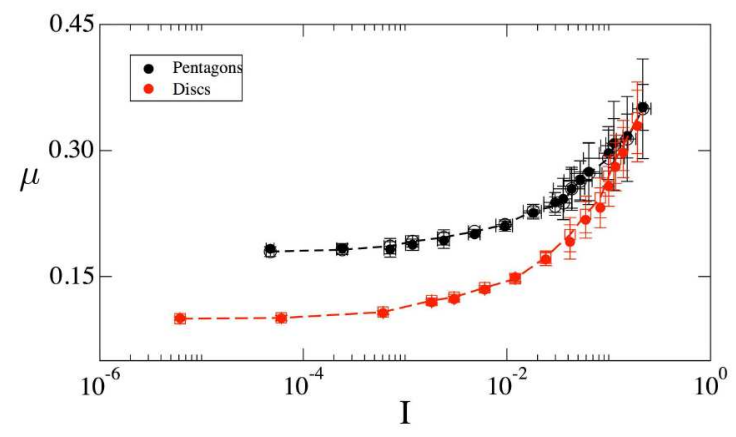

Fig. 10. (Colour online) Macroscopic friction coefficient $\mu$ (black squares for pentagons and red circles for discs) together with its harmonic approximation (eq. (15)) (dashed lines), versus $I$.

normal force tensors $[10,15]$ :

$$
\begin{aligned}
F_{i j} & =\frac{1}{N_{c}} \sum_{c \in V} n_{i}^{c} n_{j}^{c}, \\
\chi_{i j}^{n} & =\frac{1}{N_{c}} \sum_{c \in V} f_{n} n_{i}^{c} n_{j}^{c},
\end{aligned}
$$

where $N_{c}$ is the total number of contact in the sample surface $V$. Note that $F_{1}+F_{2}=1$ and $\chi_{1}^{n}+\chi_{2}^{n}=\left\langle f_{n}\right\rangle$, where the indices 1 and 2 refer to the eigenvalues of each tensor. The contact anisotropy is then given by $a_{c}=2\left(F_{1}-F_{2}\right)$ and the force chain anisotropy by $a_{f n}=2\left(\chi_{1}-\chi_{2}\right) /\left(\chi_{1}+\right.$ $\left.\chi_{2}\right)-a_{c}$. Those anisotropy parameters directly lead to an evaluation of the stress tensor. From (2), through an approach repeatedly used in previous studies $[10,15,30$, $67,68]$, one may deduce:

$$
\mu=\frac{1}{2}\left(a_{c}\left|\sin 2 \theta_{c}\right|+a_{n}\left|\sin 2 \theta_{n}\right|\right) .
$$

Relation (15) neglects quadratic terms in the anisotropy parameters, assumed sufficiently small, and also ignores a possible anisotropic angular distribution of the branch vector length. In a first approximation, as $\theta_{c}$ and $\theta_{n}$ are both close to $135^{\circ}$, except for disks at large $I$ values, one may replace $\left|\sin 2 \theta_{c}\right|$ and $\left|\sin 2 \theta_{n}\right|$ by 1 . Relation (15) is compared to measured values of $\mu$ in fig. 10, showing good agreement throughout the range of $I$. Thus eq. (15), a good approximation of $\mu$ for all values of $I$, reveals the distinct origins of the macroscopic friction.

Figure 11(b) plots $a_{c}$ and $a_{n}$ as functions of $I$. The force anisotropy parameter $a_{n}$ declines and tends to a constant value, whereas the contact anisotropy $a_{c}$ is an increasing function of $I$. Thus, by virtue of eq. (15), $\mu$ increases mainly due to the increase of $a_{c}$. The increase of $a_{c}$ is due to the loss of particle connectivity, in particular in the extension direction [69]. Normal force anisotropy $a_{n}$ reflects how force chains, in the absence of friction, are increasingly destabilized by particle inertia. It is also remarkable that at low $I$ values $a_{n}$ prevails, whereas $a_{c}$ dominates above $I>10^{-3}$, highlighting the distinct mechanical or geometrical origins of $\mu$ with respect to
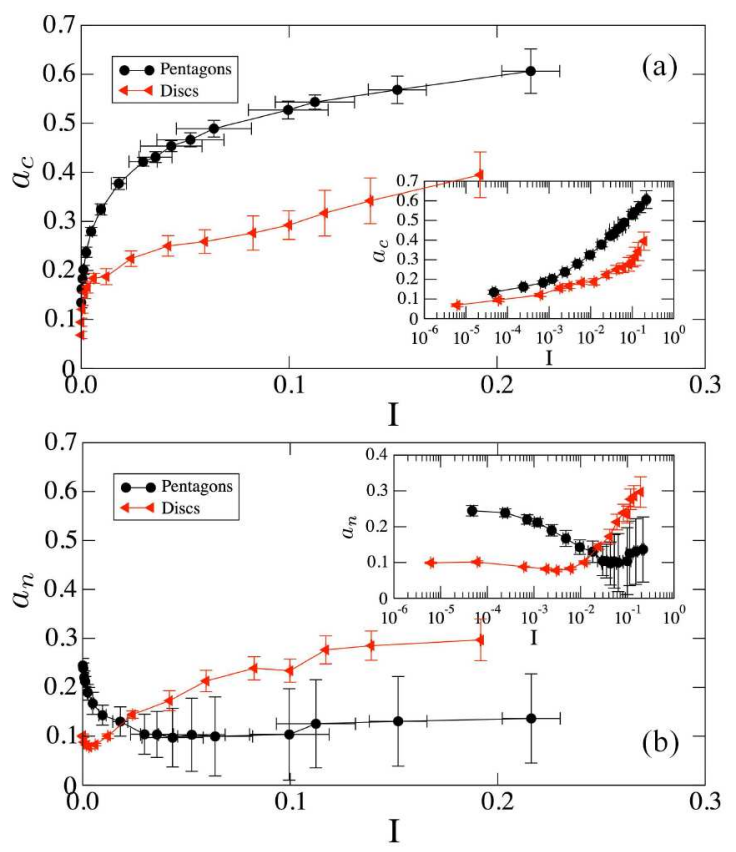

Fig. 11. (Colour online) Evolution of contact (a) and force (b) anisotropy parameters with $I$. Both insets are log-linear plots.

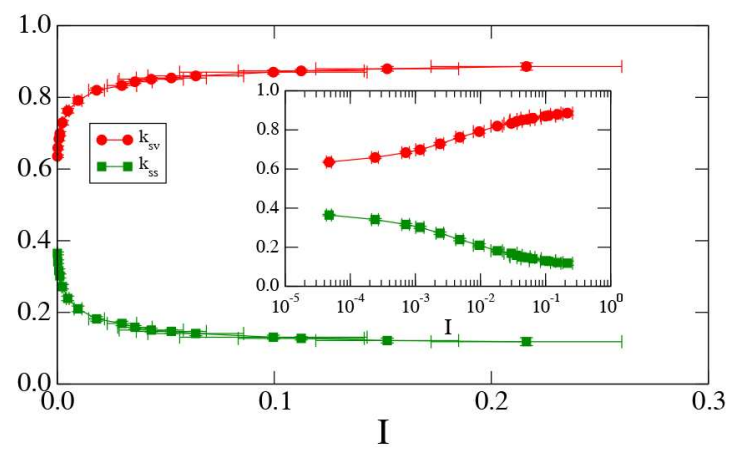

Fig. 12. (Colour online) Proportion of $s s$ and $s v$ contacts versus $I$ on linear (main plot) or logarithmic (inset) scale.

$I$. The higher value of $a_{n}$ when $I \rightarrow 0$ is attributed to side-side contacts which capture the strong force chains $[15,41,42,68]$.

\subsection{Clusters of side-side contacts}

In this section we investigate the role of side-vertex (sv) and side-side (ss) contacts and their respective $I$ dependent contributions to the overall texture and force transmission. Figure 12 shows the proportions $k_{s v}$ and $k_{s s}$ of $s s$ and $s v$ contacts as functions of $I$.

Regardless of $I$, sv contacts prevail in the contact network. In particular, $k_{s s}$, constant as $I<10^{-3}$, and declines to a constant value $k_{s s}=0.1$ at $I=10^{-2}$, which correspond also to the point where the normal force anisotropy ceases to decline and remains constant. Now, considering the contact part of eq. (2), and restricting the 

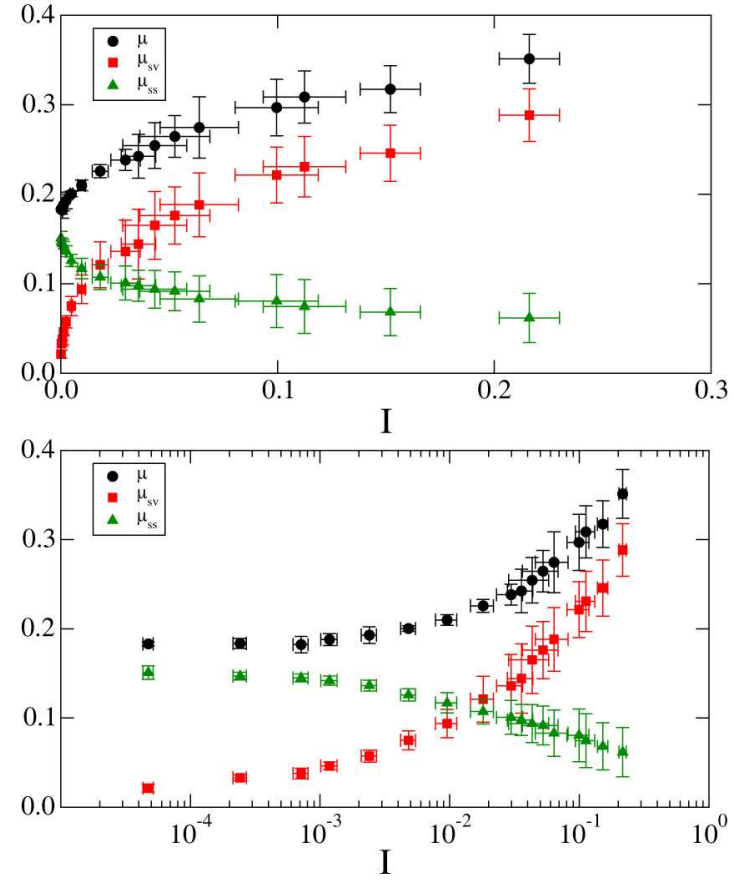

Fig. 13. (Colour online) Total and partial macroscopic friction coefficient $\mu$ for side-vertex $(s v)$ and side-side $(s s)$ contacts as functions of $I$.

summation to each contact type, one may partition the stress tensor as a sum of partial stress tensors as follows:

$$
\sigma=\sigma_{s s}+\sigma_{s v}
$$

where $\boldsymbol{\sigma}_{s s}$ and $\boldsymbol{\sigma}_{s v}$ represent the stresses carried by each contact types. Figure 13 shows the variation of corresponding contributions $\mu_{\alpha}=q_{\alpha} / p$ to the macroscopic friction, where $\alpha$ denoting contact type $s s$ and $s v$. Interestingly, below $I=10^{-3}, \mu \simeq \mu_{s s}$ whereas $\mu_{s v} \simeq 0$ showing that, in this range of $I$, a small number of $s s$-contact is at the origin of $\mu$. Such variations of $k_{s s}$ and $\mu_{s s}$ raise the issue of how those contacts are organized within our samples of frictionless pentagons.

We define clusters of particles joined by ss contacts. All particles having at least one $s s$-contact belong to one such cluster. Two clusters are disjoint if they have no common ss contact, i.e. when the boundary particles of one cluster are not connected or connected only by side-vertex contacts with the other cluster. Figure 14 shows the clusters at four values of $I$, with adjacent clusters represented with different colors. At low $I$ values most particles belong to a cluster and they seem to be all interconnected through $s v$-contacts. Note that no cluster spans the whole packing, showing that the side-side contacts do not percolate. The size of clusters declines for growing $I$, and at the largest values of $I$ they are mainly composed of only two or three particles.

These observations are presented in quantitative form in fig. 15, showing the variations with $I$ of the number of clusters, $N_{c}$, and of $\left\langle N_{p}\right\rangle_{n}$ and $\left\langle N_{p}\right\rangle_{w}$, their number or mass-averaged size, respectively. The proportion $X_{c}$ of

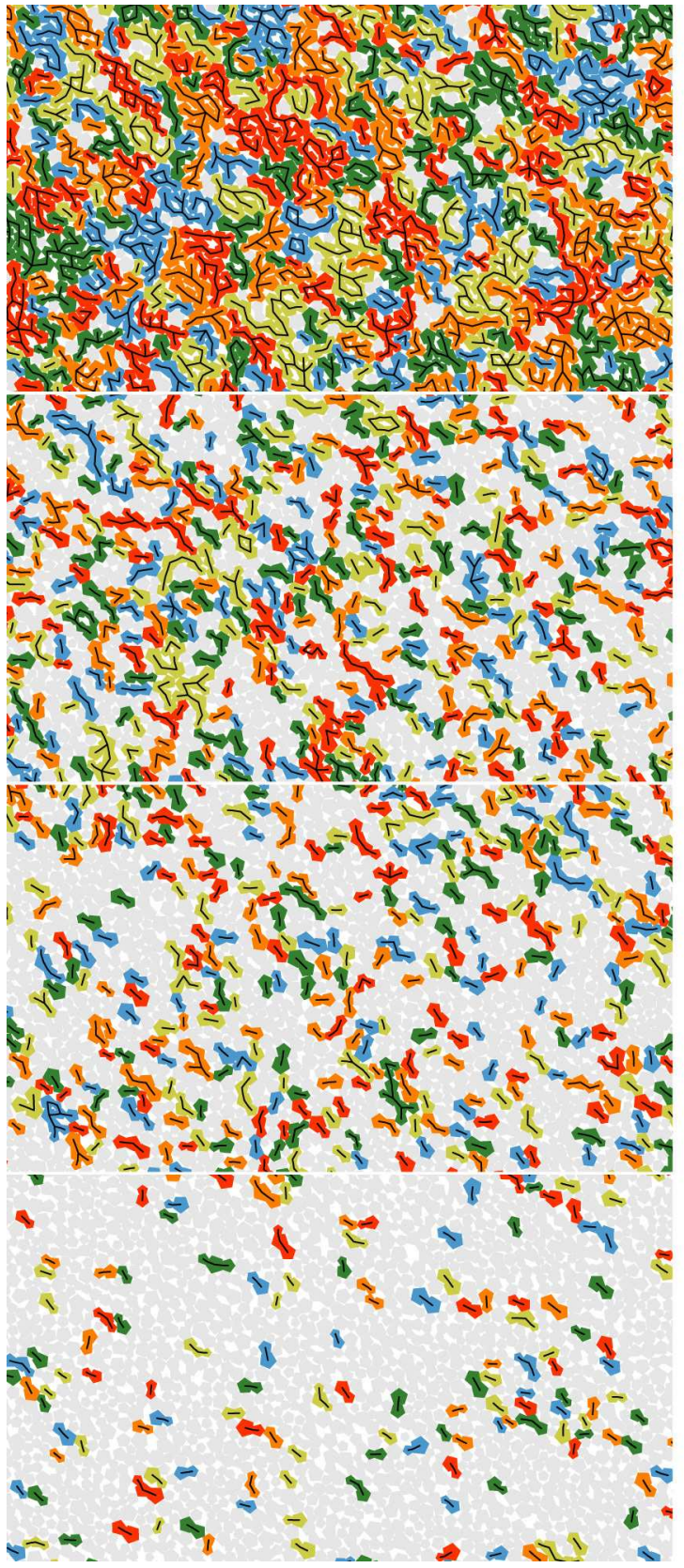

Fig. 14. (Colour online) Clusters of particles at $I=4 \cdot 10^{-5}$ (a), $I=10^{-3}$ (b), $I=10^{-2}$ (c) and $I=0.25$ (d) Disjoint clusters are represented in different colors (green, orange, purple and cardinal red). The side-side contacts are marked by a red line joining the centers of their partner particles. Others particles are in gray.

particles belonging to a cluster is

$$
X_{c}=\frac{N_{c}}{N}\left\langle N_{p}\right\rangle_{n}
$$

$X_{c}$ reaches about $77 \%$ in the quasistatic limit, and declines under growing $I$. Correlatively, both average cluster sizes decline under growing $I$. For $I>10^{-2}$ most clusters are composed of only two particles. The very moderate value of $\left\langle N_{p}\right\rangle_{w}$ in the quasistatic limit signals the absence of 

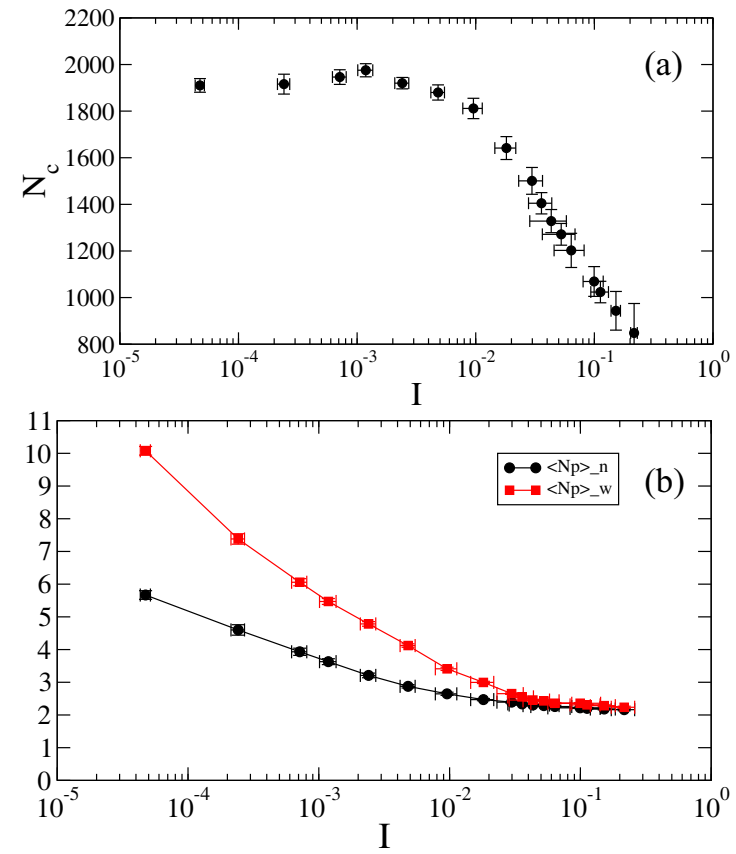

Fig. 15. (Color online) Total number $N_{c}$ of clusters (a) and number- and mass-averaged cluster sizes $\left\langle N_{p}\right\rangle_{n, w}$ (b) versus $I$. Both insets show a log-linear representation.

a percolation tendency as $I \rightarrow 0$. Interestingly, the coordination number of side-side contacts within clusters, $z_{2} / X_{c} \simeq 1.81$, with $z_{2}=z_{2}^{*}\left(1-P_{0}\right)$, is near 2 , which characterizes loopless connected structures. More precisely, the number $N^{s s}$ of grains within clusters of $s s$ contacts is related to the number of loops $L^{s s}$, the number $N_{c}$ of clusters and the number $N_{b}$ of $s s$ bonds as

$$
N^{s s}+L^{s s}=N_{b}+N_{c},
$$

a general identity for arbitrary graphs, which directly entails

$$
1+\frac{L^{s s}}{N^{s s}}=\frac{z_{2}}{2 X_{c}}+\frac{1}{\left\langle N_{p}\right\rangle_{n}}
$$

Relation (19) would reduce to the statement that the coordination number of $s s$ contacts in clusters, $\frac{z_{2}}{X_{c}}$ would be equal to 2 , if there existed only one, very large and loopless cluster. In the actual pentagon assembly sheared at low $I$, eq. (19) shows that loops of ss contacts are quite scarce, with $L^{s s} / N^{s s} \simeq 0.005$. Only a few such loops appear in fig. 14, and comparable observations could be made in the static packings of ref. [25]. The essentially loopless structure of the clusters and their size explains their rheological importance, commented above in connection with eq. (16), despite the relatively small number of $s s$ contacts. The clusters are capable of transmitting mechanical constraints on the orientation of the pentagons over distances of $5-10$ diameters.

\section{Conclusions, remarks and perspectives}

The numerical investigation of macroscopic and microstructural properties of assemblies of frictionless pen- tagon or disks in normal stress controlled shear flow, carried out over the range of inertial numbers $6.10^{-6} \leq I \leq$ 0.2 confirms the results of ref. [36] and extends them to the inertial flow regime at larger values of $I$. The macroscopic friction coefficient does not vanish and is sensitive to the grain shape in the quasistatic limit. For both particle shapes, the solid fraction is as large in quasistatic shear flow as in equilibrated solid packs under isotropic pressure, i.e., equal to the random close packing ( $\mathrm{RCP}$ ) value, as previously noted for assemblies of identical spheres in $3 \mathrm{D}[34,35]$, and different types of frictionless disk polydispersities in 2D [37]. This absence of dilatancy contrasts with the properties of frictional grains, for which the critical state - in the sense of soil mechanics - of the slowly sheared material is systematically less dense than in the RCP state. This absence of dilatancy for frictionless particles, whereas frictional ones need to dilate in shear flow, is, according to a number of recent theoretical [70], numerical [71,72], and experimental [73] studies at the origin of the discontinuous shear thickening behavior of some dense suspensions. Our observation that assemblies of frictionless angular grains should not dilate under quasistatic shear therefore supports the plausibility of such a microscopic origin of discontinuous shear thickening in suspensions of non-spherical particles.

For growing $I$, as the friction coefficient $\mu$ increases and the solid fraction $\nu$ decreases, both $I$ dependences might be described by power laws, with possibly identical exponents for disks and pentagons (near 0.5 for $\mu$, and near 0.7 for $\nu$ ). The difference between circular and angular grains seems to fade out for growing $I$, but this apparent similarity hides significant micromechanical differences. The contact network depletion, as $I$ increases, is much slower for angular particles. $\mu$ can be expressed as a simple formula adding fabric and force anisotropies parameters. While both contributions are similar throughout the $I$ interval for disks, in pentagon assemblies the fabric anisotropy dominates at large $I$, and it is almost entirely responsible for the increase of $\mu$ with $I$; the force anisotropy dominates at small $I$. Gathering in clusters the particles sharing side-side contacts, we observe those clusters, which are typically loopless, filamentary structures, to carry the major part of the shear stress at low $I$ values, and to transmit orientational constraints over limited, but significant distances in the quasistatic limit - a feature that could be responsible for the difference of internal friction between pentagons and disks.

More detailed kinematic studies investigating the rearrangements of such structures would be needed. The behavior of frictionless granular assemblies under arbitrary load directions will be the subject of future work in order to gain a better knowledge of the mechanical properties of such granular systems under a small enough stress deviator (before yielding).

\section{Author contribution statement}

The simulations, curves and analysis were done by the first author. The last and first author wrote the paper. 
The three authors proposed analysis tools and connections with the literature.

\section{References}

1. D.J. Cumberland, R.J. Crawford, The Packing of Particles (Elsevier, Amsterdam, 1987).

2. T. Aste, D. Weaire, The Pursuit of Perfect Packing (Institute of Physics Publishing, Bristol, 2000).

3. C. O'Hern, L.E. Silbert, A.J. Liu, S.R. Nagel, Phys. Rev. E 68, 011306 (2003).

4. A. Donev, S. Torquato, F.H. Stillinger, Phys. Rev. E 71, 011105 (2005).

5. I. Agnolin, J.-N. Roux, Phys. Rev. E 76, 061302 (2007).

6. A. Donev, F.H. Stillinger, P.M. Chaikin, S. Torquato, Phys. Rev. Lett. 92, 255506 (2004).

7. A. Donev, I. Cisse, D. Sachs, E.A. Variano, F.H. Stillinger, R. Connelly, S. Torquato, P.M. Chaikin, Science 303, 990 (2004).

8. W. Man, A. Donev, F.H. Stillinger, M.T. Sullivan, W.B. Russel, D. Heeger, S. Inati, S. Torquato, P.M. Chaikin, Phys. Rev. Lett. 94, 198001 (2005).

9. S. Sacanna, L. Rossi, A. Wouterse, A.P. Philipse, J. Phys.: Condens. Matter 19, 376108 (2007).

10. E. Azéma, F. Radjaï, Phys. Rev. E 81, 051304 (2010).

11. S. Torquato, Y. Jiao, Phys. Rev. E 80, 041104 (2009).

12. K.C. Smith, M. Alam, T.S. Fisher, Phys. Rev. E 82, 051304 (2010).

13. J. Baker, A. Kudrolli, Phys. Rev. E 82, 061304 (2010).

14. Y. Jiao, F.H. Stillinger, S. Torquato, Phys. Rev. E 81, 041304 (2010).

15. E. Azéma, F. Radjaï, F. Dubois, Phys. Rev. E 87, 062203 (2013).

16. E. Azéma, F. Radjaï, B. Saint-Cyr, J.-Y. Delenne, P. Sornay, Phys. Rev. E 87, 052205 (2013).

17. S. Torquato, T.M. Truskett, P.G. Debenedetti, Phys. Rev. Lett. 84, 2064 (2000).

18. P. Chaudhuri, L. Berthier, S. Sastry, Phys. Rev. Lett. 104, 165701 (2010).

19. J.-N. Roux, Phys. Rev. E 61, 6802 (2000).

20. A. Donev, R. Connelly, F.H Stillinger, S. Torquato, Phys. Rev. E 75, 051304 (2007).

21. M. Wyart, S.R. Nagel, T.A. Witten, Europhys. Lett. 72, 486 (2005).

22. C. Voivret, F. Radjai, J.-Y. Delenne, M.S. El Youssoufi, Phys. Rev. Lett. 102, 178001 (2009).

23. T.-T. Ng, Mech. Mater. 41, 748 (2009).

24. K. Szarf, G. Combe, P. Villard, Powder Technol. 208, 279 (2011).

25. D.-H. Nguyen, E. Azéma, F. Radjaï, P. Sornay, Phys. Rev. E 91, 032203 (2015).

26. J. Katagiri, T. Matsushima, Y. Yamada, Granular Matter 16, 891 (2014).

27. R. Kawamoto, E. Ando, G. Viggiana, J.E. Andrade, J. Mech. Phys. Solids 111, 375 (2018).

28. F. da Cruz, S. Emam, M. Prochnow, J.-N. Roux, F. Chevoir, Phys. Rev. E 72, 021309 (2005).

29. T. Hatano, Phys. Rev. E 75, 060301(R) (2007).

30. E. Azéma, F. Radjaï, Phys. Rev. Lett. 112, 078001 (2014).

31. J.-N. Roux, F. Chevoir, Dimensional Analysis and Control Parameters, in Discrete-Element Modeling of Granular Materials, edited by F. Radjaï, F. Dubois, (Wiley, 2011) pp. 199-232.
32. H. Troadec, F. Radjai, S. Roux, J.-C. Charmet, Phys. Rev. E 66, 041305 (2002)

33. G. Combe, J.-N. Roux, Phys. Rev. Lett. 85, 3628 (2000).

34. P.-E. Peyneau, J.-N. Roux, Phys. Rev. E 78, 041307 (2008).

35. P.-E. Peyneau, J.-N. Roux, Phys. Rev. E 78, 011307 (2008).

36. E. Azéma, F. Radjaï, J.-N. Roux, Phys. Rev. E 91, 010202(R) (2015).

37. N. Estrada, Phys. Rev. E 94, 062903 (2016).

38. D. Vågberg, P. Olsson, S. Teitel, Phys. Rev. E 93, 052902 (2016).

39. N. Estrada, W. Oquendo, Phys. Rev. E 96, 042907 (2017).

40. O. Reynolds, Philos. Mag. 20, 469 (1885).

41. E. Azéma, F. Radjai, R. Peyroux, G. Saussine, Phys. Rev. E 76, 011301 (2007).

42. E. Azéma, N. Estrada, F. Radjaï, Phys. Rev. E 86, 041301 (2012).

43. N. Estrada, E. Azéma, F. Radjai, A. Taboada, Phys. Rev. E 84, 011306 (2011).

44. CEGEO, B. Saint-Cyr, K. Szarf, C. Voivret, E. Azéma, V. Richefeu, J.-Y. Delenne, G. Combe, C. Nouguier-Lehon, P. Villard, P. Sornay, M. Chaze, F. Radjai, EPL 98, 44008 (2012).

45. J.-J. Moreau, Eur. J. Mech. A Solids 13, 93 (1994).

46. M. Jean, Comput. Methods Appl. Mech. Eng. 177, 235 (1999).

47. J.-J. Moreau, An introduction to unilateral dynamics, in Novel Approaches in Civil Engineering, Lect. Notes Appl. Comput. Mech., Vol. 14, edited by M. Frémond, F. Maceri (Springer-Verlag, Berlin, 2004) pp. 1-46.

48. F. Radjaï, V. Richefeu, Mech. Mater. 41, 715 (2009).

49. Farhang Radjai, Frédéric Dubois (Editors), Discrete Numerical Modeling of Granular Materials (Wiley-ISTE, New-York, March 2011) ISBN: 978-1-84821-260-2.

50. F. Radjai, Physics of Dry Granular Media (Kluwer Academic Publishers, Dordrecht/Boston/London, 1997) Chapt. "Multicontacts dynamics", p. 305.

51. G. Saussine, C. Cholet, P.E. Gautier, F. Dubois, C. Bohatier, J.J. Moreau, Comput. Methods Appl. Mech. Eng. 195, 2841 (2006).

52. L. Staron, F. Radjai, Phys. Rev. E 72, 041308 (2005).

53. S. Deboeuf, O. Dauchot, L. Staron, A. Mangeney, J.-P. Vilotte, Phys. Rev. E 72, 051305 (2005).

54. E. Azéma, F. Radjaï, Phys. Rev. E 85, 031303 (2012).

55. B. Saint-Cyr, C. Voivret, J.-Y. Delenne, F. Radjai, P. Sornay, Phys. Rev. E 84, 041302 (2011).

56. E. Azéma, F. Radjaï, R. Peyroux, V. Richefeu, G. Saussine, Eur. Phys. J. E 26, 327 (2008).

57. E. Azéma, Y. Descantes, N. Roquet, J.-N. Roux, F. Chevoir, Phys. Rev. E 86, 031303 (2012).

58. A. Taboada, K.J. Chang, F. Radjai, F. Bouchette, J. Geophys. Res. 110, B09202 (2005).

59. C. Voivret, F. Radjai, J.-Y. Delenne, M.S. El Youssoufi, Phys. Rev. E 76, 021301 (2007).

60. GDR-MiDi, Eur. Phys. J. E 14, 341 (2004).

61. P. Jop, Y. Forterre, O. Pouliquen, Nature 441, 727 (2006).

62. Y. Forterre, O. Pouliquen, Annu. Rev. Fluid Mech. 40, 1 (2008).

63. Bruno Andreotti, Yoel Forterre, Olivier Pouliquen, Granular Media: Between Fluid and Solid (Cambridge University Press, 2013). 
64. J.-J. Moreau, Numerical investigation of shear zones in granular materials, in Friction, Arching, Contact Dynamics, edited by D.E. Wolf, P. Grassberger (World Scientific, Singapore, 1997) pp. 233-247.

65. P.-E. Peyneau, Étude du comportement et du compactage de pâtes granulaires par simulation numérique discrète discrète, $\mathrm{PhD}$ Thesis, Ecole Nationale des Ponts et Chaussées, France, 2009.

66. D. Vågberg, P. Olsson, S. Teitel, Phys. Rev. E 95, 012902 (2017).

67. L. Rothenburg, R.J. Bathurst, Géotechnique 39, 601 (1989).
68. E. Azéma, F. Radjai, G. Saussine, Mech. Mater. 41, 721 (2009).

69. F. Radjai, J.-Y. Delenne, E. Azéma, S. Roux, Granular Matter 14, 259 (2012).

70. M. Wyart, M. Cates, Phys. Rev. Lett. 112, 098302 (2014).

71. R. Seto, R. Mari, J.F. Morris, M.M. Denn, Phys. Rev. Lett. 111, 218301 (2013).

72. D. Vågberg, P. Olsson, S. Teitel, Phys. Rev. E 95, 052903 (2017).

73. A. Clavaud, X. Bérut, B. Metzger, Y. Forterre, Proc. Natl. Acad. Sci. U.S.A. 114, 5147 (2017). 